\title{
METRIC SUBREGULARITY AND CONSTRAINT QUALIFICATIONS FOR CONVEX GENERALIZED EQUATIONS IN BANACH SPACES
}

\author{
XI YIN ZHENG ${ }^{\dagger}$ AND KUNG FU NG Z $^{\ddagger}$
}

\begin{abstract}
Several notions of constraint qualifications are generalized from the setting of convex inequality systems to that of convex generalized equations. This is done and investigated in terms of the coderivatives and the normal cones, and thereby we provide some characterizations for convex generalized equations to have the metric subregularity. As applications, we establish formulas of the modulus of calmness and provide several characterizations of the calmness. Extending the classical concept of extreme boundary, we introduce a notion of recession cores of closed convex sets. Using this concept, we establish global metric subregularity (i.e. error bound) results for generalized equations.
\end{abstract}

Key words. metric subregularity, calmness, constraint qualification, normal cone, coderivative, recession core, generalized extreme point

AMS subject classifications. 90C31, 90C25, 49J52, 46B20

1. Introduction. Let $X$ and $Y$ be Banach spaces and $F: X \rightarrow 2^{Y}$ a closed multifunction. Following Dontchev and Rockafellar [7], the multifunction $F$ is said to be metrically subregular at $a$ for $b \in F(a)$ if there exists $\tau \in[0,+\infty)$ such that

$$
d\left(x, F^{-1}(b)\right) \leq \tau d(b, F(x)) \text { for all } x \text { close to } a .
$$

The metric subregularity has been already studied by many authors under various names (cf. $[2,15,20,24,30])$. Let $A$ be a closed subset of $X$ and $b$ be a given point in $Y$. Consider the generalized equation with constraint

$$
b \in F(x) \text { subject to } x \in A \text {, }
$$

which includes most of systems in optimization. Let $S$ denote the solution set of (GEC), that is, $S=\{x \in A: b \in F(x)\}$. We say that (GEC) is metrically subregular at $a \in S$ if there exists $\tau \in(0, \infty)$ such that

$$
d(x, S) \leq \tau(d(b, F(x))+d(x, A)) \text { for all } x \text { close to } a .
$$

When $F(x)=[f(x),+\infty), b=0$ and $A=X,($ GEC) reduces to the inequality system $f(x) \leq 0$ and (1.2) means that this inequality has a local error bound at $a$. Another

*This research was supported by a postdoctoral fellowship scheme and a direct grant (CUHK),and an Earmarked Grant from the Research Grant Council of Hong Kong. Research of the first author was also supported by the National Natural Science Foundation of P. R. China (Grant No. 10361008) and the Natural Science Foundation of Yunnan Province, China (Grant No. 2003A0002M).

${ }^{\dagger}$ Department of Mathematics, Yunnan University, Kunming 650091, P. R. China (xyzheng@ynu.edu.cn).

${ }^{\ddagger}$ Department of Mathematics, The Chinese University of Hong Kong, Shatin, New Territory, Hong Kong (kfng@math.cuhk.edu.hk). 
special case is when $F(x)=[f(x),+\infty)$ and $b=\inf \{f(x): x \in A\}$. In this case, (GEC) reduces to the following optimization problem

$$
\min f(x) \text { subject to } x \in A
$$

and (1.2) means that $a$ is weak sharp minimum of (OP). Error bounds and weak sharp minima have important applications in mathematical programming and have been extensively studied (cf. [3,17,18,20,30,31]). In this paper, we mainly study the metric subregularity of (GEC) in the case when $F$ and $A$ are convex.

The notion of the basic constraint qualification (BCQ) of systems of continuous convex inequalities plays an important role in convex optimization and has been studied by many researchers (see, e.g., [11, pp.307-309] and [18,19]). Dropping the continuity assumption and adopting the singular subdifferential, the authors [32] introduced and discussed the generalized BCQ and the strong BCQ. Very recently, $\mathrm{Hu}$ [12] further studied the generalized BCQ and the strong BCQ. In Section 3, in terms of the coderivative, we extend the concept of the generalized BCQ and the strong BCQ to cover the case of generalized equation with constraint (GEC). Using the BCQ and the strong $\mathrm{BCQ}$, we provide several characterizations of the metric subregularity of (GEC).

A stronger condition is the metric regularity of a multifunction that has been well studied in variational analysis (see $[13,15,21,28]$ and references therein). Explicitly, $F$ is metrically regular at $a$ for $b \in F(a)$ if there exists $\tau \in(0,+\infty)$ such that

$$
d\left(x, F^{-1}(y)\right) \leq \tau d(y, F(x)) \text { for all }(x, y) \text { close to }(a, b) .
$$

It is well-known, as the Robinson-Ursescu theorem, that (1.3) holds if $F$ is a closed convex multifunction and $b \in \operatorname{int}(F(X))$. Under the assumption that both $X$ and $Y$ are finite dimensional, Mordukhovich [21] proved that $F$ is metrically regular at $a$ for $b \in F(a)$ if and only if $D^{*} F(a, b)^{-1}(0)=\{0\}$; moreover

$$
\inf \{\tau:(1.3) \text { holds }\}=\left\|D^{*} F(a, b)^{-1}\right\|^{+}=\limsup \left\|D^{*} F(x, y)^{-1}\right\|^{+},
$$

where $D^{*} F(a, b)$ is the coderivative of $F$ at $(a, b)$ and

$$
\left\|D^{*} F(a, b)^{-1}\right\|^{+}=\sup _{x^{*} \in B_{X^{*}}} \sup _{y^{*} \in D^{*} F(a, b)^{-1}\left(x^{*}\right)}\left\|y^{*}\right\| .
$$

To the best of our knowledge, no one has considered duality formulas similar to (1.4) for the modulus of the metric subregularity. In Section 3, we provide such formulas under the convexity assumption but no finite dimension assumption.

Similar to relationship between Aubin's pseudo-Lipschitz property and the metric regularity, the calmness is related very closely to the metric subregularity. In Section 4, as applications of results obtained in Section 3, we consider the calmness of convex multifunctions. We establish formulas of the modulus of the calmness and 
present several characterizations of the calmness in terms of the normal cone and the coderivative. In this section, we also provide characterizations of the strong calmness. Reducing to special kinds of convex multifunctions such as that recently considered by Henrion and Jourani in [8], our approach sheds light on some existing results on the calmness; in fact Corollary 4.1 provides a version that is sharper than the main result in [8].

The notion of the extreme point set of a convex set is very useful in convex analysis. In Section 5, as an extension of an extreme point set, we introduce and discuss the notion of a recession core. In terms of recession cores, we study the global metric subregularity. In particular, we show that (GEC) is globally metrically subregular if and only if (GEC) has the $\tau$-strong BCQ at each point of some recession core of the solution set $S$ for some $\tau \in(0,+\infty)$. When the solution set $S$ is a polyhedron, we obtain a sharp result that (GEC) is globally metrically subregular if and only if (GEC) has the BCQ at each point of some recession core of $S$. This implies in particular that if the graph of $F$ is a polyhedron then (GEC) is always globally metrically subregular; thus we improve the classical Hoffman result on error bound for linear inequality systems.

2. Preliminaries. Throughout this paper, we assume that $X$ and $Y$ are Banach spaces. Let $A$ be a closed convex subset of $X$. For $a \in A$, we use $T(A, a)$ to denote the tangent cone of $A$ at $a$ in the sense of convex analysis. Thus $v \in T(A, a)$ if and only if there exist a sequence $\left\{a_{n}\right\}$ in $A$ and a sequence $\left\{t_{n}\right\}$ of positive numbers convergent to 0 such that $\frac{a_{n}-a}{t_{n}}$ converges to $v$. We denote by $N(A, a)$ the normal cone of $A$ at $a$, that is,

$$
N(A, a):=\left\{x^{*} \in X^{*}:\left\langle x^{*}, x-a\right\rangle \leq 0 \text { for all } x \in A\right\} .
$$

Let $F: X \rightarrow 2^{Y}$ be a multifunction and denote by $\operatorname{Gr}(F)$ the graph of $F$, that is,

$$
\operatorname{Gr}(F):=\{(x, y) \in X \times Y: y \in F(x)\} .
$$

As usual, $F$ is said to be closed (resp. convex) if $\operatorname{Gr}(F)$ is a closed (resp. convex) subset of $X \times Y$. It is known that $F$ is convex if and only if

$$
t F\left(x_{1}\right)+(1-t) F\left(x_{2}\right) \subset F\left(t x_{1}+(1-t) x_{2}\right) \quad \forall x_{1}, x_{2} \in X \text { and } \forall t \in[0,1] .
$$

Let $F$ be a closed convex multifunction and $(x, y) \in \operatorname{Gr}(F)$. The tangent derivative $D F(x, y)$ of $F$ at $(x, y)$ is defined by

$$
D F(x, y)(u)=\{v \in Y:(u, v) \in T(\operatorname{Gr}(F),(x, y))\} \quad \forall u \in X
$$

(cf. [1]). Let $D^{*} F(x, y)$ denote the coderivative of $F$ at $(x, y)$, which is defined by

$$
D^{*} F(x, y)\left(y^{*}\right):=\left\{x^{*} \in X^{*}:\left(x^{*},-y^{*}\right) \in N(\operatorname{Gr}(F),(x, y))\right\} \quad \forall y^{*} \in Y^{*}
$$

(cf. [21,22,28]). Let $G: X \rightarrow 2^{Y}$ be a sublinear multifunction (i.e., $\operatorname{Gr}(G)$ is a convex cone in $X \times Y)$. Let $B_{X}$ denote the closed unit ball of $X$. As in Dontchev, Lewis and 
Rockafellar [6], the outer norm and the inner norm of $G$ are respectively defined as follows

$$
\|G\|^{+}=\sup _{x \in B_{X}} \sup _{y \in G x}\|y\| \quad \text { and } \quad\|G\|^{-}:=\sup _{x \in B_{X}} \inf _{y \in G x}\|y\|
$$

with the usual convention that the infimum and the supremum over an empty set are $+\infty$ and $-\infty$, respectively. For a convex cone $C$ in $X$, let $\left\|\left.G\right|_{C}\right\|^{+}$and $\left\|\left.G\right|_{C}\right\|^{-}$be respectively defined by

$$
\left\|\left.G\right|_{C}\right\|^{+}:=\sup _{x \in B_{X} \cap C} \sup _{y \in G x}\|y\| \text { and }\left\|\left.G\right|_{C}\right\|^{-}:=\sup _{x \in B_{X} \cap C} \inf _{y \in G x}\|y\| .
$$

We denote by $\operatorname{bd}(A)$ the topological boundary of a subset $A$ of $X$. The following lemma is known (cf. [23, Proposition 1.3] or [24, Lemma 2.1]) and useful for us.

Lemma 2.1. Let $X$ be a Banach space and $A$ a closed convex nonempty subset of $X$. Then, for any $\beta \in(0,1)$ and any $x \in X \backslash A$ there exist $z \in \operatorname{bd}(A)$ and $x^{*} \in N(A, z)$ with $\left\|x^{*}\right\|=1$ such that

$$
\beta\|x-z\|<d(x, A) \text { and } \beta\|x-z\|<\left\langle x^{*}, x-z\right\rangle .
$$

3. BCQ, strong BCQ and metric subregularity. Throughout this section, we assume that $F: X \rightarrow 2^{Y}$ is a closed convex multifunction, $A$ is a closed convex subset of $X$ and that $b$ is a given point in $X$. Recall that $S=\{x \in A: b \in F(x)\}$ is the solution set of the corresponding generalized equation with constraint (GEC).

Recently, in dealing with the inequality defined by a proper lower semicontinuous convex function, the authors [32] introduced and discussed the generalized BCQ and the strong BCQ.

In terms of the coderivative replacing the subdifferential and the singular subdifferential, we can extend the concept of the generalized BCQ and the strong BCQ to the case of generalized equation with constraint (GEC). Explicitly, we say that (GEC) has the BCQ at $a \in S$ if

$$
N(S, a)=D^{*} F(a, b)\left(Y^{*}\right)+N(A, a)
$$

and (GEC) has the strong BCQ at $a \in S$ if there exists $\tau \in(0,+\infty)$ such that

$$
N(S, a) \cap B_{X^{*}} \subset \tau\left(D^{*} F(a, b)\left(B_{Y^{*}}\right)+B_{X *} \cap N(A, a)\right) .
$$

The following Theorem establishes relationship between the metric subregularity and the strong BCQ.

THEOREM 3.1. Let $a \in S$. Then, generalized equation (GEC) is metrically subregular at $a$ if and only if there exist $\tau, \delta \in(0,+\infty)$ such that $(G E C)$ has the strong $B C Q$ at all points in $\operatorname{bd}(S) \cap B(a, \delta)$ with the same constant, where $B(a, \delta)$ denotes the open ball with center a and radius $\delta$. 
Proof. Suppose that (GEC) is metrically subregular at $a$. Then there exist $\tau, \delta \in$ $(0,+\infty)$ such that $(1.2)$ holds. For any $(x, y) \in X \times Y$, let $\|(x, y)\|_{\tau}:=\frac{\tau+1}{\tau}\|x\|+\|y\|$. Then $\|\cdot\|_{\tau}$ is a norm on $X \times Y$ inducing the product topology, and the unit ball of the dual space of $\left(X \times Y,\|\cdot\|_{\tau}\right)$ is $\left(\frac{\tau}{\tau+1} B_{X^{*}}\right) \times B_{Y^{*}}$. We claim that

$$
d(x, S) \leq \tau\left(d_{\|\cdot\|_{\tau}}((x, y), \operatorname{Gr}(F))+\|y-b\|+d(x, A)\right) \quad \forall(x, y) \in B\left(a, \frac{\delta}{2}\right) \times Y,
$$

where the distance $d_{\|\cdot\|_{\tau}}$ is with respect to the norm $\|\cdot\|_{\tau}$. Suppose to the contrary that (3.3) does not hold. Then there exists $\left(x_{0}, y_{0}\right) \in B\left(a, \frac{\delta}{2}\right) \times Y$ such that

$$
d\left(x_{0}, S\right)>\tau\left[d_{\|\cdot\|_{\tau}}\left(\left(x_{0}, y_{0}\right), \operatorname{Gr}(F)\right)+\left\|y_{0}-b\right\|+d\left(x_{0}, A\right)\right] .
$$

It follows that there exists $u \in X$ such that

$$
d\left(x_{0}, S\right)>\tau\left(\frac{\tau+1}{\tau}\left\|u-x_{0}\right\|+d\left(y_{0}, F u\right)+\left\|y_{0}-b\right\|+d\left(x_{0}, A\right)\right),
$$

and hence

$$
d\left(x_{0}, S\right)>\left\|u-x_{0}\right\|+\tau(d(b, F u)+d(u, A)) .
$$

Noting that

$$
\|u-a\| \leq\left\|u-x_{0}\right\|+\left\|x_{0}-a\right\|<d\left(x_{0}, S\right)+\left\|x_{0}-a\right\| \leq 2\left\|x_{0}-a\right\|<\delta,
$$

it follows from (1.2) and the triangle inequality that

$$
d\left(x_{0}, S\right)>\left\|u-x_{0}\right\|+d(u, S) \geq d\left(x_{0}, S\right)
$$

which is a contradiction. Hence (3.3) holds. We will establish the necessary part by showing that

$$
N(S, z) \cap B_{X^{*}} \subset \tau\left(D^{*} F(z, b)\left(B_{Y^{*}}\right)+B_{X^{*}} \cap N(A, z)\right) \quad \forall z \in B\left(a, \frac{\delta}{2}\right) \cap S .
$$

To do this, let $z \in S \cap B\left(a, \frac{\delta}{2}\right)$ and $x^{*} \in B_{X^{*}} \cap N(S, z)$. Since $A$ is convex, $B_{X^{*}} \cap$ $N(S, z)=\partial d(\cdot, S)(z)$ (cf. [4, Theorem 1]). Thus,

$$
\left\langle x^{*}, x-z\right\rangle \leq d(x, S)-d(z, S)=d(x, S) \text { for all } x \in X .
$$

It follows from (3.3) that

$\left.\left\langle x^{*}, x-z\right\rangle \leq \tau\left(d_{\|\cdot\|_{\tau}}(x, y), \operatorname{Gr}(F)\right)+\|y-b\|+d(x, A)\right) \quad \forall(x, y) \in B\left(z, \frac{\delta}{2}-\|z-a\|\right) \times Y$.

Together with the convexity of $F$ and $A$, this implies that $\left(\frac{x^{*}}{\tau}, 0\right) \in \partial \phi(z, b)$, where $\phi$ is the convex function defined by

$$
\phi(x, y):=d_{\|\cdot\|_{\tau}}((x, y), \operatorname{Gr}(F))+\|y-b\|+d(x, A) \quad \forall(x, y) \in X \times Y .
$$


Noting that

$$
\partial d_{\|\cdot\|_{\tau}}(\cdot, \operatorname{Gr}(F))(z, b) \subset N(\operatorname{Gr}(F),(z, b)),
$$

it follows from [5, Proposition 2.3.2] that

$$
\left(\frac{x^{*}}{\tau}, 0\right) \in N(\operatorname{Gr}(F),(z, b))+\{0\} \times B_{Y^{*}}+\left(B_{X^{*}} \cap N(A, z)\right) \times\{0\} .
$$

This implies that $x^{*} \in \tau\left(D^{*} F(z, b)\left(B_{Y^{*}}\right)+B_{X^{*}} \cap N(A, z)\right)$, and hence that (3.4) holds as required to show.

Conversely, suppose that there exist $\tau^{\prime}, \delta^{\prime} \in(0,+\infty)$ such that (GEC) has the strong BCQ at each point of $\operatorname{bd}(S) \cap B\left(a, \delta^{\prime}\right)$ with the constant $\tau^{\prime}$. Let $x \in B\left(a, \frac{\delta^{\prime}}{2}\right) \backslash S$. Then, $d(x, S) \leq\|x-a\|<\frac{\delta^{\prime}}{2}$. Let $\beta \in\left(\frac{2 d(x, S)}{\delta^{\prime}}, 1\right)$. Then, by Lemma 2.1 there exists $u \in \operatorname{bd}(S)$ and $x^{*} \in N(S, u)$ with $\left\|x^{*}\right\|=1$ such that $\beta\|x-u\| \leq d(x, S)$ and

$$
\beta\|x-u\| \leq\left\langle x^{*}, x-u\right\rangle .
$$

Thus, $\|x-u\|<\frac{\delta^{\prime}}{2}$. Hence $\|u-a\| \leq\|u-x\|+\|x-a\|<\delta^{\prime}$, and so (GEC) has the strong BCQ at $u$ with the constant $\tau$. Therefore, there exists $y^{*} \in B_{Y^{*}}$, $x_{1}^{*} \in D^{*} F(u, b)\left(y^{*}\right)$ and $x_{2}^{*} \in B_{X^{*}} \cap N(A, u)=\partial d(\cdot, A)(u)$ (by [4,Theorem 1]) such that $x^{*}=\tau^{\prime}\left(x_{1}^{*}+x_{2}^{*}\right)$. By the convexity of $F$ and $A$, one has

$$
\left\langle x_{1}^{*}, x-u\right\rangle \leq\left\langle y^{*}, y-b\right\rangle \quad \forall y \in F(x) \text { and }\left\langle x_{2}^{*}, x-u\right\rangle \leq d(x, A)-d(u, A)=d(x, A)
$$

Hence,

$$
\left\langle x^{*}, x-u\right\rangle \leq \tau^{\prime}\left(\left\langle y^{*}, y-b\right\rangle+d(x, A)\right) \leq \tau^{\prime}(\|y-b\|+d(x, A)) \quad \forall y \in F(x)
$$

This and (3.5) imply that $\beta\|x-u\| \leq \tau^{\prime}(d(b, F(x))+d(x, A))$. It follows from $u \in S$ that $\beta d(x, S) \leq \tau^{\prime}(d(b, F(x))+d(x, A))$. Since $\beta$ can be arbitrarily close to $1, d(x, S) \leq \tau^{\prime}(d(b, F(x))+d(x, A))$. This shows that (GEC) is metrically subregular at $a$. This completes the proof.

Theorem 3.1 recaptures some earlier results dealing with only numerical valued functions. Let $f: X \rightarrow R \cup\{+\infty\}$ be a proper lower semicontinuous convex function. When $F(x)=[f(x),+\infty), b=0$ and $A=X$, Theorem 3.1 was obtained in [32]. A slightly earlier result is due to Burke and Deng who showed in [3, Theorem 5.2] that if $X$ is a Hilbert space, $F(x)=[f(x),+\infty), b=\inf _{x \in X} f(x)$ and $A=X$, then (GEC) is metrically subregular at $a$ if and only if there exists $\tau \in[0,+\infty)$ such that

$$
N(S, x) \cap B_{X^{*}} \subset \tau \mathrm{cl}^{*}(\partial f(a)),
$$

where $\mathrm{cl}^{*}$ denotes the weak ${ }^{*}$ closure.

Remark 3.1. Let $\tau(F, a, b ; A):=\inf \{\tau>0:(1.2)$ holds $\}$. For $u \in S$, let $\gamma(F, u, b ; A):=\inf \{\tau>0:($ GEC) has the strong BCQ at $u$ with the constant $\tau\}$. 
By the proof of Theorem 3.1, one can see that

$$
\tau(F, a, b ; A)=\limsup _{u \stackrel{\operatorname{bd}(S)}{\longrightarrow} a} \gamma(F, u, b ; A) \geq \gamma(F, a, b ; A) .
$$

In general, (GEC) is not necessarily metrically subregular at $a$ if (GEC) has the strong BCQ only at a (see [32, Example 2]). But, when $S$ is assumed to be "locally conical" at $a$, Theorem 3.1 and (3.6) can be sharpened. To do this, we need the following lemma.

LEMmA 3.1. Let $s_{1}, s_{2} \in S$ be such that $\left\langle u^{*}, s_{1}\right\rangle=\left\langle u^{*}, s_{2}\right\rangle$. Then, $u^{*} \in D^{*} F\left(s_{1}, b\right)\left(B_{Y^{*}}\right)+B_{X^{*}} \cap N\left(A, s_{1}\right) \Leftrightarrow u^{*} \in D^{*} F\left(s_{2}, b\right)\left(B_{Y^{*}}\right)+B_{X^{*}} \cap N\left(A, s_{2}\right)$.

Proof. Obviously we need only to prove one direction of the implications, say " $\Rightarrow$ ". Let

$$
\psi(x, y):=\|y-b\|+d(x, A)+\delta_{\mathrm{Gr}(F)}(x, y) \text { for all }(x, y) \in X \times Y,
$$

where $\delta_{\operatorname{Gr}(F)}$ denotes the indicator function of $\operatorname{Gr}(F)$. It follows from [4, Theorem 1] and [5, Proposition 2.3.2] that

$$
\partial \psi(s, b)=\{0\} \times B_{Y^{*}}+\left(B_{X^{*}} \cap N(A, s)\right) \times\{0\}+N(\operatorname{Gr}(F),(s, b)) \quad \forall s \in S .
$$

Suppose that $u^{*} \in D^{*} F\left(s_{1}, b\right)\left(B_{Y^{*}}\right)+B_{X^{*}} \cap N\left(A, s_{1}\right)$. Then, by (3.7), one has $\left(u^{*}, 0\right) \in \partial \psi\left(s_{1}, b\right)$. Hence,

$$
\left\langle u^{*}, x-s_{1}\right\rangle \leq \psi(x, y)-\psi\left(s_{1}, b\right) \text { for all }(x, y) \in X \times Y \text {. }
$$

Since $\left\langle u^{*}, s_{1}\right\rangle=\left\langle u^{*}, s_{2}\right\rangle$ and $\psi\left(s_{1}, b\right)=\psi\left(s_{2}, b\right)=0$,

$$
\left\langle u^{*}, x-s_{2}\right\rangle \leq \psi(x, y)-\psi\left(s_{2}, b\right) \text { for all }(x, y) \in X \times Y \text {. }
$$

Therefore, $\left(u^{*}, 0\right) \in \partial \psi\left(s_{2}, b\right)$. It follows from (3.7) that $u^{*} \in D^{*} F\left(s_{2}, b\right)\left(B_{Y^{*}}\right)+$ $B_{X^{*}} \cap N\left(A, s_{2}\right)$. This shows that the implication " $\Rightarrow$ " holds. The proof is completed.

Theorem 3.2. Let $a \in S$. Suppose that there exist a cone $C$ and a neighborhood $V$ of a such that $S \cap V=(a+C) \cap V$. Then

$$
\tau(F, a, b ; A)=\gamma(F, a, b ; A) .
$$

Consequently, (GEC) is metrically subregular at a if and only if (GEC) has the strong $B C Q$ at $a$.

Proof. In view of (3.6), we need only to show that

$$
\limsup _{\substack{\operatorname{bd}(S) \\ u \stackrel{(}{\longrightarrow} a}} \gamma(F, u, b ; A) \leq \gamma(F, a, b ; A) .
$$


Let $\delta>0$ be such that $B(a, \delta) \subset V$. To prove (3.8), it suffices to show that for any $u \in S \cap B(a, \delta)$,

$$
\gamma(F, u, b ; A) \leq \gamma(F, a, b ; A) .
$$

We first show that

$$
N(S, u) \subset N(S, a) \text { for all } u \in S \cap B(a, \delta) \text {. }
$$

To do this, let $u \in S \cap B(a, \delta)$ and $x^{*} \in N(S, u)$. Noting that $V$ is a neighborhood of $u$, we have

$$
N(S, u)=N(S \cap V, u)=N((a+C) \cap V, u)=N(a+C, u) .
$$

Choosing $c_{u} \in C$ such that $u=a+c_{u}$, it follows that

$$
\left\langle x^{*}, a+c_{u}\right\rangle=\sup \left\{\left\langle x^{*}, a+c\right\rangle: c \in C\right\} .
$$

Since $C$ is a cone, it follows that $\left\langle x^{*}, c_{u}\right\rangle=0$ and hence

$$
\left\langle x^{*}, u\right\rangle=\left\langle x^{*}, a\right\rangle=\sup \left\{\left\langle x^{*}, a+c\right\rangle: c \in C\right\} .
$$

This implies that $x^{*} \in N(a+C, a)=N(S, a)$. Therefore, (3.10) holds. Since (3.9) trivially holds if $\gamma(F, a, b ; A)=+\infty$, we assume henceforth that $\gamma(F, a, b ; A)<+\infty$. Let $r \in(\gamma(F, a, b ; A),+\infty)$. Then,

$$
B_{X^{*}} \cap N(S, a) \subset r\left(D^{*} F(a, b)\left(B_{Y^{*}}\right)+B_{X^{*}} \cap N(A, a)\right) .
$$

Let $u \in S \cap B(a, \delta)$ and $x^{*} \in B_{X^{*}} \cap N(S, u)$. By (3.10), one has

$$
x^{*} \in r\left(D^{*} F(a, b)\left(B_{Y^{*}}\right)+B_{X^{*}} \cap N(A, a)\right) .
$$

It follows from (3.11) and Lemma 3.1 that $x^{*} \in r\left(D^{*} F(u, b)\left(B_{Y^{*}}\right)+B_{X^{*}} \cap N(A, u)\right)$. Therefore,

$$
B_{X^{*}} \cap N(S, u) \subset r\left(D^{*} F(u, b)\left(B_{Y^{*}}\right)+B_{X^{*}} \cap N(A, u)\right) .
$$

This implies that $\gamma(F, u, b ; A) \leq r$. Letting $r \rightarrow \gamma(F, a, b ; A)$, one sees that (3.9) holds. The proof is completed.

Remark 3.2. If the solution set $S$ is a polyhedron then for each $a \in S$ there exist a cone $C$ and a neighborhood $V$ of $a$ such that $S \cap V=(a+C) \cap V$; in fact, in this case we can choose $C$ to be the tangent cone of $S$ at $a$.

TheOREM 3.3. Let $a \in S$,

$$
\tau_{1}:=\inf \{\tau>0: d(x, a+T(S, a)) \leq \tau(d(b, F(x))+d(x, A)) \text { for all } x \text { close to } a\}
$$


and

$$
\tau_{2}:=\inf \{\tau>0: d(h, T(S, a)) \leq \tau(d(0, D F(a, b)(h))+d(h, T(A, a))) \quad \forall h \in X\} .
$$

Then

$$
\tau_{1}=\tau_{2}=\gamma(F, a, b ; A)
$$

Moreover,

$$
\tau_{2}<+\infty \Longrightarrow T(S, a)=T(A, a) \cap D F(a, b)^{-1}(0) .
$$

Consequently, $(G E C)$ has the strong $B C Q$ at a if and only if the sublinear generalized equation (with constraint)

$$
0 \in D F(a, b)(x) \text { subject to } x \in T(A, a)
$$

is metrically subregular at 0 .

Proof. We first show that $\tau_{1}=\tau_{2}$. Let $h \in X, y \in D F(a, b)(h), u \in T(A, a)$ and $\varepsilon>0$. Then, there exists $t>0$ small enough such that

$$
(h, y) \in \frac{\operatorname{Gr}(F)-(a, b)}{t}+\varepsilon B_{X} \times \varepsilon B_{Y} \text { and } u \in \frac{A-a}{t}+\varepsilon B_{X} .
$$

Therefore, there exists $z \in B_{X}$ such that

$$
b+t y \in F(a+t h+t \varepsilon z)+t \varepsilon B_{Y} \text { and } a+t u \in A+t \varepsilon B_{X} .
$$

This implies that

$$
d(b, F(a+t h+t \varepsilon z)) \leq t\|y\|+t \varepsilon \text { and } d(a+t h+t \varepsilon z, A) \leq t\|h-u\|+2 t \varepsilon .
$$

Considering an arbitrary $\tau>\tau_{1}$ and noting that $t>0$ is small enough, it follows that

$$
\begin{aligned}
\tau t(\|y\|+\|h-u\|+3 \varepsilon) & \geq d(a+t h+t \varepsilon z, a+T(S, a)) \\
& \geq d(t h, T(S, a))-t \varepsilon \\
& =t d(h, T(S, a))-t \varepsilon
\end{aligned}
$$

where the last equality holds because $T(S, a)$ is a cone. Therefore,

$$
d(h, T(S, a)) \leq \tau(d(0, D F(a, b)(h))+d(h, T(A, a)))+(3 \tau+1) \varepsilon .
$$

Letting $\varepsilon \rightarrow 0$ and $\tau \rightarrow \tau_{1}$, one has

$$
d(h, T(S, a)) \leq \tau_{1}(d(0, D F(a, b)(h))+d(h, T(A, a))) .
$$

Hence $\tau_{2} \leq \tau_{1}$.

Conversely, by the convexity of $F$, one has

$$
\operatorname{Gr}(F)-(a, b) \subset T(\operatorname{Gr}(F),(a, b))=\operatorname{Gr}(D F(a, b)) .
$$


Then, for any $x \in X, F(x)-b \subset D F(a, b)(x-a)$, and so

$$
d(0, D F(a, b)(x-a)) \leq d(b, F(x))
$$

On the other hand, the convexity of $A$ implies that

$$
d(x-a, T(A, a)) \leq d(x-a, A-a) \leq d(x, A) .
$$

Hence, for any $x \in X$,

$d(x-a, T(S, a)) \leq \tau_{2}(d(0, D F(a, b)(x-a))+d(x-a, T(A, a))) \leq \tau_{2}(d(b, F(x))+d(x, A))$.

Therefore $\tau_{1} \leq \tau_{2}$ and so $\tau_{1}=\tau_{2}$ is shown. Next we show that $\gamma(F, a, b ; A)=\tau_{2}$. By the definition of $\tau_{2}$, we have

$$
d(x, T(S, a)) \leq \tau_{2}(d(0, D F(a, b)(x))+d(x, T(A, a))) \text { for all } x \in X .
$$

In the case when $\tau_{2}<+\infty$, this implies that $T(A, a) \cap D F(a, b)^{-1}(0) \subset T(S, a)$ and hence (3.12) is seen to hold as the converse inclusion is easily verified by the convexity of $F$ and $A$. From (3.12) it is straightforward to verify that

$$
\gamma(F, a, b ; A)=\gamma(D F(a, b), 0,0 ; T(A, a)) \text { and } \tau_{2}=\tau(D F(a, b), 0,0 ; T(A, a)) .
$$

This and Theorem 3.2 imply that $\tau_{2}=\gamma(F, a, b ; A)$. In the case when $\tau_{2}=+\infty$, suppose to the contrary that $\tau_{2} \neq \gamma(F, a, b ; A)$. Then, $\gamma(F, a, b ; A)<+\infty$. Let $x \in X \backslash T(S, a)$ and $\beta \in(0,1)$. By Lemma 2.1 there exist $u \in T(S, a)$ and $x^{*} \in$ $N(T(S, a), u)$ such that

$$
\left\|x^{*}\right\|=1 \text { and }\left\langle x^{*}, x-u\right\rangle \geq \beta\|x-u\| .
$$

Noting that $N(T(S, a), u) \subset N(T(S, a), 0)$ (because $T(S, a)$ is a closed convex cone), it follows that $x^{*} \in N(T(S, a), 0)=N(S, a)$ and $\left\langle x^{*}, u\right\rangle=0$. Take a fixed $\eta$ in $(\gamma(F, a, b ; A), \infty)$. Then there exist $y^{*} \in \eta B_{Y^{*}}, x_{1}^{*} \in D^{*} F(a, b)\left(y^{*}\right)$ and $x_{2}^{*} \in \eta B_{X^{*}} \cap$ $N(A, a)$ such that $x^{*}=x_{1}^{*}+x_{2}^{*}$. Equipping the product space $X \times Y$ with norm $\|(x, y)\|_{\eta}=\frac{\eta}{1+\eta}\|x\|+\|y\|$ for all $(x, y) \in X \times Y$ and noting that the unit ball of the dual space of $\left(X \times Y,\|\cdot\|_{\eta}\right)$ is $\left(\frac{\eta+1}{\eta} B_{X^{*}}\right) \times B_{Y^{*}}$, it follows from (2.1) and the convexity of $D F(a, b)$ and $A$ that

$$
\begin{aligned}
\frac{1}{\eta}\left(x_{1}^{*},-y^{*}\right) & \in N(\operatorname{Gr}(F),(a, b)) \cap\left(\left(\frac{\eta+1}{\eta} B_{X^{*}}\right) \times B_{Y^{*}}\right) \\
& =N(\operatorname{Gr}(D F(a, b)),(0,0)) \cap\left(\left(\frac{\eta+1}{\eta} B_{X^{*}}\right) \times B_{Y^{*}}\right) \\
& =\partial d_{\|\cdot\|_{\eta}}(\cdot, \operatorname{Gr}(D F(a, b)))(0,0)
\end{aligned}
$$

and

$$
\frac{1}{\eta} x_{2}^{*} \in B_{X^{*}} \cap N(A, a)=B_{X^{*}} \cap N(T(A, a), 0)=\partial d(\cdot, T(A, a))(0) .
$$


Therefore,

$$
\frac{1}{\eta}\left\langle x_{1}^{*}, x\right\rangle \leq d_{\|\cdot\|_{\eta}}((x, 0), \operatorname{Gr}(D F(a, b)) \leq d(0, D F(a, b)(x))
$$

and $\frac{1}{\eta}\left\langle x_{2}^{*}, x\right\rangle \leq d(x, T(A, a))$. Noting that $\left\langle x^{*}, u\right\rangle=0$, it follows from (3.13) that

$$
\frac{\beta\|u-x\|}{\eta} \leq d(0, D F(a, b)(x))+d(x, T(A, a)) .
$$

Therefore, $\frac{\beta d(x, T(S, a))}{\eta} \leq d(0, D F(a, b)(x))+d(x, T(A, a))$. Letting $\beta \rightarrow 1$, one has

$$
d(x, T(S, a)) \leq \eta(d(0, D F(a, b)(x))+d(x, T(A, a))) .
$$

This contradicts $\tau_{2}=+\infty$. The proof is completed.

Let $\phi: X \rightarrow R \cup\{+\infty\}$ be a proper lower semicontinuous convex function. Consider the special case when $A=X$ and $F(x)=[\phi(x),+\infty)$ for all $x \in X$. In this case, $N(A, x)=\{0\}$ for any $x \in A$. For $a \in \operatorname{dom}(\phi)$, let $\partial^{\infty} \phi(a)$ denote the singular subdifferential of $\phi$ at $a$, namely $\partial^{\infty} \phi(a)=D^{*} F(a, \phi(a))(0)$. It is easy to verify from the convexity of $\phi$ that $\operatorname{dom}\left(D^{*} F(a, \phi(a))\right) \subset R_{+}$. Thus, noting that

$$
D^{*} F(a, \phi(a))(1)=\partial \phi(a) \text { and } \partial \phi(a)=\partial \phi(a)+\partial^{\infty} \phi(a)
$$

and adopting the convention that $R_{+} \partial \phi(a)$ and $[0,1] \partial \phi(a)$ are $\{0\}$ if $\partial \phi(a)=\emptyset$, one has

$$
\begin{aligned}
D^{*} F(a, \phi(a))(R) & =D^{*} F(a, \phi(a))(0) \bigcup D^{*} F(a, \phi(a))\left(R_{+} \backslash\{0\}\right) \\
& =D^{*} F(a, \phi(a))(0) \bigcup R_{+} D^{*} F(a, \phi(a))(1) \\
& =\partial^{\infty} \phi(a)+R_{+} \partial \phi(a)
\end{aligned}
$$

and

$$
D^{*} F(a, \phi(a))([-1,1])=\partial^{\infty} \phi(a)+[0,1] \partial \phi(a) .
$$

Therefore, our definitions of the BCQ and the strong BCQ for generalized equations are respectively natural generalization of the BCQ and the strong BCQ of a convex inequality system (cf. [17-19, 32]). Thus, Theorems 3.1 and 3.3 extend Theorems 2.2 and 2.3 in [32] from the setting of a convex inequality to that of a convex generalized equation with constraint.

Since the strong BCQ implies the BCQ, the following proposition shows that the converse also holds in some interesting cases.

Proposition 3.1. Let $a \in S$ and suppose that $N(S, a)$ is a polyhedron in a finite dimensional subspace of $X^{*}$. Then (GEC) has the BCQ at a if and only if it has the strong $B C Q$ at a. 
Proof. We need only to show the necessity part. Suppose that (GEC) has the BCQ at $a$. It suffices to show that there exists $\tau>0$ such that

$$
B_{X^{*}} \cap N(S, a) \subset \tau\left(D^{*} F(a, b)\left(B_{Y^{*}}\right)+B_{X^{*}} \cap N(A, a)\right) .
$$

Let $E$ be a finite dimensional subspace of $X^{*}$ such that $N(S, a) \subset E$. Let

$$
L:=N(S, a) \cap-N(S, a)
$$

namely $L$ is the largest subspace contained in $N(S, a)$. Take a subspace $L^{\perp}$ of $E$ such that

$$
L \cap L^{\perp}=\{0\} \text { and } E=L+L^{\perp} .
$$

Since $N(S, a)$ is a polyhedral cone in $E$, by [27, Theorem 19.1] there exists a polyhedron cone $C \subset L^{\perp}$ containing no lines such that

$$
N(S, a)=C+L
$$

On the other hand, $\operatorname{dim}(E)<\infty$ and (3.15) imply that there exists $\delta \in(0,+\infty)$ such that $B_{X^{*}} \cap(C+L) \subset \delta\left(B_{X^{*}} \cap C+B_{X^{*}} \cap L\right)$. It follows from (3.16) that

$$
B_{X^{*}} \cap N(S, a) \subset \delta\left(B_{X^{*}} \cap C+B_{X^{*}} \cap L\right)
$$

Since $L$ is a finite dimensional space, there exist $l_{1}, \cdots, l_{m} \in L$ such that

$$
B_{X}^{*} \cap L \subset \operatorname{co}\left(l_{1}, \cdots, l_{m}\right)
$$

Take $c_{1}, \cdots, c_{n} \in C$ such that $C=R^{+} \operatorname{co}\left(c_{1}, \cdots, c_{n}\right)$ and $0 \notin \operatorname{co}\left(c_{1}, \cdots, c_{n}\right)$ (because $C$ is a finite dimensional polyhedron cone containing no lines). Without loss of generality we assume that $B_{X^{*}} \cap \operatorname{co}\left(c_{1}, \cdots, c_{n}\right)=\emptyset$. We note that

$$
B_{X}^{*} \cap C \subset \operatorname{co}\left(0, c_{1}, \cdots, c_{n}\right)
$$

By (3.16) and the BCQ assumption, there exist

$$
\left\{y_{1}^{*}, \cdots, y_{n}^{*}, \tilde{y}_{1}^{*}, \cdots, \tilde{y}_{m}^{*}\right\} \subset Y^{*} \text { and }\left\{a_{1}^{*}, \cdots, a_{n}^{*}, \tilde{a}_{1}^{*}, \cdots, \tilde{a}_{m}^{*}\right\} \subset N(A, a)
$$

such that

$$
c_{i} \in D^{*} F(a, b)\left(y_{i}^{*}\right)+a_{i}^{*}, 1 \leq i \leq n \text { and } l_{j} \in D^{*} F(a, b)\left(\tilde{y}_{j}^{*}\right)+\tilde{a}_{j}^{*}, 1 \leq j \leq m .
$$

Let $\kappa:=\max _{1 \leq i \leq n, 1 \leq j \leq m}\left\|y_{i}^{*}\right\|+\left\|a_{i}^{*}\right\|+\left\|\tilde{y}_{j}^{*}\right\|+\left\|\tilde{a}_{j}^{*}\right\|$. It follows from (3.18) and (3.19) that

$$
B_{X^{*}} \cap C+B_{X^{*}} \cap L \subset \kappa\left(D^{*} F(a, b)\left(B_{Y^{*}}\right)+N(A, a) \cap B_{X^{*}}\right)
$$

This and (3.17) imply that (3.14) holds with $\tau=\delta \kappa$. 
Corollary 3.1. Let $f_{1}, \cdots, f_{n}: X \rightarrow R \cup\{+\infty\}$ be proper lower semicontinuous convex functions and consider generalized equation (GEC) with $A=X, Y=R^{n}$, $b=\left(b_{1}, \cdots, b_{n}\right) \in R^{n}$ and $F$ being defined by

$$
F(x)=\left(f_{1}(x), \cdots, f_{n}(x)\right)+R_{+}^{n} \text { for all } x \in X
$$

Suppose that each $f_{i}$ is differentiable at $a \in S$. Then, for the said generalized equation, the $B C Q$ and the strong $B C Q$ are equivalent at $a$.

Proof. In view of Proposition 3.1, it suffices to show that

$$
D^{*} F(a, b)\left(R^{n}\right)=R_{+} \operatorname{co}\left\{f_{i}^{\prime}(a): i \in J(a)\right\},
$$

where $J(a):=\left\{1 \leq i \leq n: f_{i}(a)=b_{i}\right\}$. To do this, we first note that $\operatorname{dom}\left(D^{*} F(a, b)\right)=$ $R_{+}^{n}$ (because each convex function $f_{i}$ is differentiable at $a$ ). Let $\left(r_{1}, \cdots, r_{n}\right) \in R_{+}^{n} \backslash\{0\}$ and $x^{*} \in D^{*} F(a, b)\left(r_{1}, \cdots, r_{n}\right)$. Then

$$
\left\langle x^{*}, x\right\rangle-\sum_{i=1}^{n} r_{i}\left(f_{i}(x)+t_{i}\right) \leq\left\langle x^{*}, a\right\rangle-\sum_{i=1}^{n} r_{i} b_{i}
$$

for any $x \in X$ and $\left(t_{1}, \cdots, t_{n}\right) \in R_{+}^{n}$. Noting that $f_{i}(a)=b_{i}$ for any $i \in J(a)$, $f_{i}(a)<b_{i}$ for any $i \notin J(a)$ and $a \in \operatorname{int}\left(\operatorname{dom}\left(f_{i}\right)\right)$ for $1 \leq i \leq n$, it follows that $r_{i}=0$ for any $i \notin J(a)$ and

$$
\left\langle x^{*}, x-a\right\rangle \leq \sum_{i \in J(a)} r_{i} f(x)-\sum_{i \in J(a)} r_{i} f_{i}(a)
$$

for all $x \in X$. This implies that $x^{*}=\sum_{i \in J(a)} r_{i} f_{i}^{\prime}(a)$. Thus, $x^{*} \in R_{+} \operatorname{co}\left\{f_{i}^{\prime}(a): i \in\right.$ $J(a)\}$. This shows that $D^{*} F(a, b)\left(R^{n}\right) \subset R_{+} \operatorname{co}\left\{f_{i}^{\prime}(a): i \in J(a)\right\}$. Conversely, let $x^{*} \in R_{+} \operatorname{co}\left\{f_{i}^{\prime}(a): i \in J(a)\right\}$. Then there exists $\left(c_{1}, \cdots, c_{n}\right) \in R_{+}^{n}$ with $c_{i}=0$ for all $i \notin J(a)$ such that $x^{*}=\sum_{i=1}^{n} c_{i} f_{i}^{\prime}(a)$. Noting that for each $i$,

$$
\left\langle c_{i} f_{i}^{\prime}(a), x-a\right\rangle \leq c_{i}\left(f_{i}(x)+t_{i}-b_{i}\right) \quad \forall x \in X \text { and } \forall t_{i} \geq 0 .
$$

it follows that $x^{*} \in D^{*} F(a, b)\left(c_{1}, \cdots, c_{n}\right)$. This shows that

$$
R_{+} \operatorname{co}\left\{f_{i}^{\prime}(a): i \in J(a)\right\} \subset D^{*} F(a, b)\left(R^{n}\right) .
$$

Hence (3.20) holds.

4. Calmness of convex multifunctions. Throughout this section, let $M$ : $Y \rightarrow 2^{X}$ be a closed convex multifunction and $A$ be a closed convex subset of $X$. Let $\bar{y} \in Y$ and $\bar{x} \in M(\bar{y}) \cap A$. Recall (cf. [8-10] and [15]) that $M$ is said to be calm at $(\bar{y}, \bar{x})$ if there exists a constant $\mathrm{E}>0$ such that

$$
d(x, M(\bar{y})) \leq L\|y-\bar{y}\| \quad \text { for all }(y, x) \in \operatorname{Gr}(M) \text { close to }(\bar{y}, \bar{x}) .
$$


More generally, $M$ is said to be calm at $(\bar{y}, \bar{x})$ over $A$ if there exists a constant $\mathrm{\iota}>0$ such that

$$
d(x, M(\bar{y}) \cap A) \leq L(\|y-\bar{y}\|+d(x, A)) \quad \text { for all }(y, x) \in \operatorname{Gr}(M) \text { close to }(\bar{y}, \bar{x}) .
$$

Let $\tilde{M}: Y \times X \rightarrow 2^{X}$ be defined by

$$
\tilde{M}(y, z)=M(y) \cap(-z+A) \text { for any }(y, z) \in Y \times X
$$

and $Y \times X$ be equipped with the norm $\|(y, z)\|=\|y\|+\|z\|$ for any $(y, z) \in Y \times X$. Then, as observed by one of the referees, (4.2) holds if and only if

$$
d(x, \tilde{M}(\bar{y}, 0)) \leq L\|(y, z)-(\bar{y}, 0)\| \text { for all }(y, z ; x) \in \operatorname{Gr}(\tilde{M}) \text { close to }(\bar{y}, 0 ; \bar{x}) .
$$

Hence, $M$ is calm at $(\bar{y}, \bar{x})$ over $A$ if and only if $\tilde{M}$ is calm at $(\bar{y}, 0 ; \bar{x})$. A more general intersection map have been studied by Klatte and Kummer [16]. Since $d(x, \emptyset)=+\infty$ and $d(x, M(\bar{y})) \leq\|x-\bar{x}\|$, it is easy to verify that (4.2) holds if and only if

$$
d(x, M(\bar{y}) \cap A) \leq L\left(d\left(\bar{y}, M^{-1}(x)\right)+d(x, A)\right) \text { for all } x \text { close to } \bar{x}
$$

Letting $b=\bar{y}$ and $F(x)=M^{-1}(x)$, it follows that (GEC), defined by the data $(F, A, b)$, is metrically subregular at $\bar{x}$ if and only if $M$ is calm at $(\bar{y}, \bar{x})$ over $A$. Thus, by Theorems 3.1 and 3.3, we have the following results.

THEOREM 4.1. The following statements are equivalent.

(i) $M$ is calm at $(\bar{y}, \bar{x})$ over $A$.

(ii) There exist $\tau, \delta \in(0,+\infty)$ such that for all $u \in B(\bar{x}, \delta) \cap \operatorname{bd}(M(\bar{y}) \cap A)$,

$$
N(M(\bar{y}) \cap A, u) \cap B_{X^{*}} \subset \tau\left(D^{*} M^{-1}(\bar{x}, \bar{y})\left(B_{Y^{*}}\right)+N(A, u) \cap B_{X^{*}}\right) .
$$

(iii) There exists $\delta \in(0,+\infty)$ such that for all $u \in \operatorname{bd}(M(\bar{y}) \cap A)$ close to $\bar{x}$, the tangent derivative $D M(\bar{y}, u)$ is calm at $(0,0)$ over $T(A, u)$ with the same constant.

Imitating the notion of the strong metric regularity (cf. [7]), we say that $M$ is strongly calm at $(\bar{y}, \bar{x})$ over $A$ if there exists $L \in[0,+\infty)$ such that

$$
\|x-\bar{x}\| \leq L(\|y-\bar{y}\|+d(x, A)) \text { for all }(y, x) \in \operatorname{Gr}(M) \text { close to }(\bar{y}, \bar{x}) .
$$

From the convexity of $M(\bar{y}) \cap A$, it is not difficult to verify that $M$ is strongly calm at $(\bar{y}, \bar{x})$ over $A$ if and only if $M(\bar{y}) \cap A=\{\bar{x}\}$ and $M$ is calm at $(\bar{y}, \bar{x})$ over $A$. Using Theorem 4.1, we can establish some characterization of the strong calmness.

COROLlary 4.1. The following statements are equivalent.

(i) $M$ is strongly calm at $(\bar{y}, \bar{x})$ over $A$.

(ii) There exists $L \in[0,+\infty)$ such that

$$
\|x-\bar{x}\| \leq L(\|y-\bar{y}\|+d(x, A)) \text { for all }(y, x) \in \operatorname{Gr}(M)
$$


(iii) The tangent derivative $D M(\bar{y}, \bar{x})$ is strongly calm at $(0,0)$ over $T(A, \bar{x})$.

(iv) $0 \in \operatorname{int}\left(D^{*} M^{-1}(\bar{x}, \bar{y})\left(Y^{*}\right)+N(A, \bar{x})\right)$.

(v) There exists $r>0$ such that

$$
r B_{X^{*}} \subset D^{*} M^{-1}(\bar{x}, \bar{y})\left(B_{Y^{*}}\right)+B_{X^{*}} \cap N(A, \bar{x}) .
$$

Proof. It is clear that (ii) $\Longrightarrow(\mathrm{i})$ and (v) $\Longrightarrow$ (iv). We show next that (i) $\Leftrightarrow(\mathrm{v})$. By the evident fact $N(\{\bar{x}\}, \bar{x})=X^{*}$ and by Theorem 4.1, we need only show that (v) $\Rightarrow M(\bar{y}) \cap A=\{\bar{x}\}$. Take an arbitrary $x \in M(\bar{y}) \cap A$ and $x^{*} \in B_{X^{*}}$ such that $\|x-\bar{x}\|=\left\langle x^{*}, x-\bar{x}\right\rangle$. By (v), there exist $y^{*} \in B_{Y^{*}}, x_{1}^{*} \in D^{*} M^{-1}(\bar{x}, \bar{y})\left(y^{*}\right)$ and $x_{2}^{*} \in N(A, \bar{x}) \cap B_{X^{*}}$ such that $r x^{*}=x_{1}^{*}+x_{2}^{*}$. Hence

$$
r\|x-\bar{x}\|=\left\langle x_{1}^{*}, x-\bar{x}\right\rangle+\left\langle x_{2}^{*}, x-\bar{x}\right\rangle .
$$

Noting that $\left\langle x_{1}^{*}, x-\bar{x}\right\rangle \leq\left\langle y^{*}, \bar{y}-\bar{y}\right\rangle=0$ and $\left\langle x_{2}^{*}, x-\bar{x}\right\rangle \leq 0$, it follows that $r\|x-\bar{x}\| \leq 0$ for any $x \in M(\bar{y}) \cap A$. This shows that $M(\bar{y}) \cap A=\{\bar{x}\}$; thus (i) $\Leftrightarrow(\mathrm{v})$.

Noting that $D^{*} M^{-1}(\bar{x}, \bar{y})=D^{*}(D M(\bar{y}, \bar{x}))^{-1}(0,0)$, we have (iii) $\Leftrightarrow(\mathrm{v})$ by $(\mathrm{i}) \Leftrightarrow(\mathrm{v})$, applied to $D M(\bar{y}, \bar{x})$ in place of $M$.

Suppose that (i) holds. Then there exists $L \in[0,+\infty)$ such that (4.4) holds. Let $(y, x)$ be an arbitrary element in $\operatorname{Gr}(M)$ and $t \in(0,1)$ be small enough such that $(t y+(1-t) \bar{y}, t x+(1-t) \bar{x})$ close enough to $(\bar{y}, \bar{x})$. By (4.4) and the convexity of $M$, one has

$$
\|t x+(1-t) \bar{x}-\bar{x}\| \leq L(\|t y+(1-t) \bar{y}-\bar{y}\|+d(t x+(1-t) \bar{x}, A)) .
$$

It follows from the convexity of $A$ and $\bar{x} \in A$ that $\|x-\bar{x}\| \leq L(\|y-\bar{y}\|+d(x, A))$. This shows that (i) $\Longrightarrow$ (ii).

It remains to show that (iv) $\Rightarrow(\mathrm{v})$. Suppose that (iv) holds. Since $N(A, x)$ and $D^{*} M^{-1}(x, \bar{y})\left(Y^{*}\right)$ are cones,

$$
X^{*}=D^{*} M^{-1}(\bar{x}, \bar{y})\left(Y^{*}\right)+N(A, \bar{x})=\bigcup_{n=1}^{\infty}\left(D^{*} M^{-1}(\bar{x}, \bar{y})\left(n B_{Y^{*}}\right)+N(A, \bar{x}) \cap n B_{X^{*}}\right) .
$$

Noting that, by the Alaoglu theorem, each set $D^{*} M^{-1}(\bar{x}, \bar{y})\left(n B_{Y^{*}}\right)+N(A, \bar{x}) \cap n B_{X^{*}}$ is weak ${ }^{*}$ closed, it follows from the well-known Baire category theorem and (iv) that

$$
0 \in \operatorname{int}\left(D^{*} M^{-1}(\bar{x}, \bar{y})\left(B_{Y^{*}}\right)+N(A, \bar{x}) \cap B_{X^{*}}\right) .
$$

Hence there exists $r>0$ such that (v) holds. The proof is completed.

Remark. In a recent paper [8] Henrion and Jourani considered the calmness of the convex multifunction $M_{0}$ of the following type

$$
M_{0}(y)=\{x \in C: f(x) \leq y\} \text { for all } y \in R,
$$


where $C$ is a closed convex subset of $X$ and $f: X \rightarrow R \cup\{+\infty\}$ is a proper lower semicontinuous convex function. In particular, as a main result, they established the following result .

Theorem HJ ([8, Theorem 3.3]). Let $M_{0}$ be defined by (4.5). Then $M_{0}$ is calm at $(0, \bar{x}) \in \operatorname{Gr}\left(M_{0}\right)$ if one of the following conditions is satisfied:

(C1) $f(\bar{x})<0$.

(C2) bd $f(\bar{x}) \cap \operatorname{bd} N(C, \bar{x}) \neq \partial f(\bar{x}) \cap N(C, \bar{x})$.

(C3) bd $f(\bar{x}) \cap \operatorname{bd} N(C, \bar{x})=\emptyset$ and $\left(\mathrm{CD}^{*}\right)$ (see [8] for the definition of condition $\left.\left(\mathrm{CD}^{*}\right)\right)$.

As observed by Henrion and Jourani $[8],(\mathrm{C} 3) \Longrightarrow(\mathrm{C} 2)$ or $(\mathrm{C} 1)$ and

$$
(\mathrm{C} 2) \Longrightarrow \operatorname{int} \partial f(\bar{x}) \cap-N(C, \bar{x}) \neq \emptyset \text { or } \partial f(\bar{x}) \cap-\operatorname{int} N(C, \bar{x}) \neq \emptyset .
$$

Hence $(\mathrm{C} 2) \Longrightarrow 0 \in \operatorname{int}(\partial f(\bar{x})+N(C, \bar{x}))$. Considering that $(\mathrm{C} 1) \Longrightarrow$ the calmness of $M_{0}$ at $(0, \bar{x})$ is an immediate consequence of the Robinson-Ursescu theorem (cf. $[25,29])$, the main part of Theorem HJ can be rewritten as follows.

Theorem $\mathbf{H} \mathbf{J}^{\prime} . M_{0}$ is calm at $(0, \bar{x}) \in \operatorname{Gr}\left(M_{0}\right)$ if

$$
f(\bar{x})=0 \text { and } 0 \in \operatorname{int}(\partial f(\bar{x})+N(C, \bar{x})) .
$$

Let $A=C, Y=R$ and $M(y)=\{x \in X: f(x) \leq y\}$ for all $y \in Y$. It is clear that $M_{0}$ is calm at $(0, \bar{x})$ if $M$ is calm at $(0, \bar{x})$ over $A$. Since

$$
\partial f(\bar{x})=D^{*} M^{-1}(\bar{x}, f(\bar{x}))(1) \subset D^{*} M^{-1}(\bar{x}, f(\bar{x}))\left(Y^{*}\right),
$$

(4.6) is stronger than (v) in Corollary 4.1. Hence Corollary 4.1 improves Theorem HJ'. Similarly, one can see that Corollary 4.1 improve [8, Theorem 4.3] when $0 \notin \operatorname{int}(C-D)$. As in the beginning of the proof of [8, Theorem 4.3], it is an immediate consequence of the Robinson-Ursescu theorem when $0 \notin \operatorname{int}(C-D)$.

The calmness modulus of $M$ at $(\bar{y}, \bar{x})$ is denoted by $\eta(M ; \bar{y}, \bar{x})$ and is defined by

$$
\eta(M ; \bar{y}, \bar{x}):=\inf \{L \in(0,+\infty):(4.1) \text { holds }\} .
$$

As applications of Theorems 3.1 and 3.2 , we establish formulas representing $\eta(M ; \bar{y}, \bar{x})$.

Theorem 4.2. $\eta(M ; \bar{y}, \bar{x})=\limsup _{\operatorname{bd}(M(\overline{)})}\left\|\left.D^{*} M(\bar{y}, u)\right|_{-N(M(\bar{y}), u)}\right\|^{-}$

Proof. Since $(4.2) \Leftrightarrow(4.3), \eta(M ; \vec{y}, \bar{x})=\tau\left(M^{-1}, \bar{x}, \bar{y} ; X\right)$. By (3.6), it suffices to show that

$$
\gamma\left(M^{-1}, u, \bar{y} ; X\right)=\left\|\left.D^{*} M(\bar{y}, u)\right|_{-N(M(\bar{y}), u)}\right\|^{-} \quad \forall u \in M(\bar{y}) .
$$

Let $u \in M(\bar{y})$ and $\tau>\gamma\left(M^{-1}, u, \bar{y} ; X\right)$. Noting that $N(X, u)=\{0\}$, one has

$$
N(M(\bar{y}), u) \cap B_{X^{*}} \subset D^{*} M^{-1}(u, \bar{y})\left(\tau B_{Y^{*}}\right) .
$$


Hence, for any $x^{*} \in N(M(\bar{y}), u) \cap B_{X^{*}}$ there exists $y^{*} \in B_{Y^{*}}$ such that $x^{*} \in$ $D^{*} M^{-1}(u, \bar{y})\left(\tau y^{*}\right)$, that is, $-\tau y^{*} \in D^{*} M(\bar{y}, u)\left(-x^{*}\right)$. It follows that

$$
\left\|\left.D^{*} M(\bar{y}, u)\right|_{-N(M(\bar{y}), u)}\right\|^{-} \leq \tau .
$$

Therefore, $\left\|\left.D^{*} M(\bar{y}, u)\right|_{-N(M(\bar{y}), u)}\right\|^{-} \leq \gamma\left(M^{-1}, u, \bar{y} ; X\right)$. To prove the converse inequality, let $\tau>\left\|\left.D^{*} M(\bar{y}, u)\right|_{-N(M(\bar{y}), u)}\right\|^{-}$and $x^{*} \in N(M(\bar{y}), u) \cap B_{X^{*}}$. Then, there exists $y^{*} \in D^{*} M(\bar{y}, u)\left(-x^{*}\right)$ such that $\left\|y^{*}\right\|<\tau$; this implies that $x^{*} \in$ $D^{*} M^{-1}(u, \bar{y})\left(\tau B_{Y^{*}}\right)$. Hence, $N(M(\bar{y}), u) \cap B_{X^{*}} \subset D^{*} M^{-1}(u, \bar{y})\left(\tau B_{Y^{*}}\right)$. It follows that $\gamma\left(M^{-1}, u, \bar{y} ; X\right) \leq \tau$. Therefore,

$$
\gamma\left(M^{-1}, u, \bar{y} ; X\right) \leq\left\|\left.D^{*} M(\bar{y}, u)\right|_{-N(M(\bar{y}), u)}\right\|^{-} .
$$

This shows that (4.7) holds.

Remark. In contrast to formula (1.4) of the modulus of the metric regularity, $\eta(M ; \bar{y}, \bar{x})$ is not necessarily equivalent to $\left\|\left.D^{*} M(\bar{y}, \bar{x})\right|_{-N(M(\bar{y}), \bar{x})}\right\|^{-}$even when $Y=$ $R^{2}$ and $X=R$ (cf. [30, Example 2]). Nevertheless, the following theorem shows an interesting case for which the equality holds.

THEOREM 4.3. Suppose that there exist a cone $C$ and a neighborhood $V$ of $\bar{x}$ such that $M(\bar{y}) \cap V=(\bar{x}+C) \cap V$. Then $\eta(M ; \bar{y}, \bar{x})=\left\|\left.D^{*} M(\bar{y}, \bar{x})\right|_{-N(M(\bar{y}), \bar{x})}\right\|^{-}$.

The proof of Theorem 4.3 is similar to that of Theorem 4.2 but using Theorem 3.2 in place of Theorem 3.1 .

5. Recession core and global metric subregularity. Let $K$ be a closed convex subset of $X$. Recall that $e \in K$ is called an extreme point of $K$ if $x_{1}=x_{2}$ whenever $e=t x_{1}+(1-t) x_{2}$ with $x_{1}, x_{2} \in K$ and $t \in(0,1)$. We denote by $\operatorname{ext}(K)$ the set of all extreme points of $K$ (usually $\operatorname{ext}(K)$ is called the extreme boundary of $K)$. Let $K^{\infty}$ denote the recession cone of $K$, that is,

$$
K^{\infty}:=\{h \in X: K+t h \subset K \text { for all } t \geq 0\}
$$

It is known that $K^{\infty}$ is a closed convex cone, and

$$
\begin{aligned}
K^{\infty} & =\left\{h \in X: x+R_{+} h \subset K \text { for some } x \in K\right\} \\
& =\left\{h \in X: \exists x_{n} \in K \text { and } \exists t_{n}>0 \text { such that } t_{n} \rightarrow 0 \text { and } t_{n} x_{n} \rightarrow h\right\} .
\end{aligned}
$$

Clearly, $K+K^{\infty}=K$. It is well known that if $K$ is a closed convex subset of $R^{n}$ containing no lines then $K=\operatorname{co}(\operatorname{ext}(K))+K^{\infty}$. As a generalization of $\operatorname{co}(\operatorname{ext}(K))$, the authors [24] introduced the concept of recession property: a convex subset $A$ of $K$ is said to have the recession property if $K=A+K^{\infty}$. Simplifying the recession property, now we can give a generalization of $\operatorname{ext}(K)$ : a subset $C$ of $K$ is said to be a recession core of $K$ if

$$
K=\operatorname{co}(C)+K^{\infty}
$$


Thus, $C$ is a recession core of $K$ if and only if $\operatorname{co}(C)$ is a subset of $K$ with recession property. Let " $\leq_{K^{\infty}}$ " denote the order induced by the cone $K^{\infty}$, that is, $x_{1} \leq_{K^{\infty}} x_{2}$ if and only if $x_{2}-x_{1} \in K^{\infty}$. Let $A$ be a subset of $X$. We say that $a \in A$ is a minimal element of $A$ with respect to " $\leq_{K^{\infty}}$ " if $a \leq_{K^{\infty}} x$ whenever $x \in A$ and $x \leq_{K^{\infty}} a$. We denote by $\operatorname{Min}\left(A, K^{\infty}\right)$ the set of all minimal elements of $A$. Let

$$
\operatorname{Lin}(K):=K^{\infty} \cap-K^{\infty} .
$$

Then

$$
\operatorname{Lin}(K)=\{h \in X: K+R h \subset K\}=\{h \in X: x+R h \subset K \text { for some } x \in K\} .
$$

Moreover, $K=K+\operatorname{Lin}(K)$ and $L \subset \operatorname{Lin}(K)$ whenever $L$ is a subspace of $X$ such that $K=K+L$. Therefore, $K$ contains no lines if and only if $\operatorname{Lin}(K)=\{0\}$.

Proposition 5.1. Let $X$ be a reflexive Banach space and $K$ a closed convex nonempty subset of $X$. Suppose that there exists a closed convex bounded set $\Theta$ such that

$$
\operatorname{Lin}(K) \cap \Theta=\emptyset \text { and } K^{\infty}=\operatorname{Lin}(K)+R_{+} \Theta .
$$

Then

$$
K=\operatorname{Min}\left(K, K^{\infty}\right)+K^{\infty}
$$

In particular, $\operatorname{Min}\left(K, K^{\infty}\right)$ is a recession core of $K$.

Proof. By the reflexivity of $X$, the bounded closed convex set $\Theta$ is weakly compact. Letting $K_{0}:=R_{+} \Theta$, it follows that $K_{0}$ is a closed convex pointed cone. Let $x$ be an arbitrary point in $K$. We claim that $K \cap\left(x-K_{0}\right)$ is bounded. If this is not the case, then there exist a sequence $\left\{s_{n}\right\}$ in $R_{+}$and a sequence $\left\{\theta_{n}\right\}$ in $\Theta$ such that $s_{n} \rightarrow \infty$ and $x-s_{n} \theta_{n} \in K$ for all $n$. Thus, for any $t>0$,

$$
x-t \theta_{n}=\left(1-\frac{t}{s_{n}}\right) x+\frac{t}{s_{n}}\left(x-s_{n} \theta_{n}\right) \in K \text { for all } n \text { large enough. }
$$

By the weak compactness of $\Theta$, without loss of generality we can assume that $\left\{\theta_{n}\right\}$ converges weakly to some $\theta \in \Theta$. Therefore, $x-t \theta \in K$ for any $t \geq 0$. This implies that $-\theta \in K^{\infty}$. On the other hand, by the second equality in (5.2), one has $\theta \in K^{\infty}$, and so $\theta \in \operatorname{Lin}(K)$. This contradicts the first equality in (5.2) and therefore $K \cap\left(x-K_{0}\right)$ must be bounded (and hence weakly compact). It follows from [14, Corollary 3.1.16]) that $\operatorname{Min}\left(K \cap\left(x-K_{0}\right), K_{0}\right) \neq \emptyset$. Take $x^{\prime} \in \operatorname{Min}\left(K \cap\left(x-K_{0}\right), K_{0}\right)$. Then $x^{\prime} \in \operatorname{Min}\left(K, K_{0}\right)$ and $x \in x^{\prime}+K_{0}$. Hence $K \subset \operatorname{Min}\left(K, K_{0}\right)+K_{0}$. Now to show (5.3), it suffices to show that $\operatorname{Min}\left(K, K_{0}\right) \subset \operatorname{Min}\left(K, K^{\infty}\right)$. Let $z \in \operatorname{Min}\left(K, K_{0}\right)$ and $y \in K$ with $y \leq_{K^{\infty}} z$. Then $z-y \in K^{\infty}$. By the second equality of (5.2), there exists $e \in \operatorname{Lin}(K)$ such that $z-y-e \in K_{0}$, that is, $y+e \leq_{K_{0}} z$. Noting that $y+e \in K$, one has that $y+e-z \in K_{0}$ and hence $y-z \in \operatorname{Lin}(K)+K_{0}=K^{\infty}$. This shows that 
$z \in \operatorname{Min}\left(K, K^{\infty}\right)$. The proof is completed.

In the case when $X$ is finite dimensional, the assumption made in (5.2) automatically holds (by $K=\operatorname{Lin}(K)+C$ and Klee's Theorem (cf. [14]), where $C$ is a closed convex pointed cone). The following example shows that the reflexivity of $X$ cannot be removed in Proposition 5.1.

Example. Let $X=l^{1}$ and $K=\left\{x=\left(t_{1}, t_{2}, \cdots\right) \in l^{1}: t_{n} \geq-n, \quad \forall n\right\}$. It is easy to verify that $\operatorname{Lin}(K)=\{0\}$ and $K^{\infty}=\left\{x=\left(t_{1}, t_{2}, \cdots\right) \in l^{1}: t_{n} \geq 0, \quad \forall n\right\}$. Let $\Theta:=\left\{x=\left(t_{1}, t_{2}, \cdots\right) \in K^{\infty}: \sum_{n=1}^{\infty} t_{n}=1\right\}$. Then, $\Theta$ is a bounded closed convex set, $\operatorname{Lin}(K) \cap \Theta=\emptyset$ and $K^{\infty}=R^{+} \Theta$. But $\operatorname{Min}\left(K, K^{\infty}\right)=\emptyset$, and hence $K \neq \operatorname{Min}\left(K, K^{\infty}\right)+K^{\infty}$. Indeed, let $x=\left(t_{1}, t_{2}, \cdots\right)$ be any point in $K$. Noting that $\sum_{n=1}^{\infty}\left|t_{n}\right|<\infty$, there exists a natural number $n_{0}$ such that $\left|t_{n}\right|<n_{0}$ for all $n \geq n_{0}$. Take $x_{0}=\left(s_{1}, s_{2}, \cdots\right)$ to satisfy $s_{n}=t_{n}$ for any $n \neq n_{0}$ and $s_{n_{0}}=-n_{0}$. It is clear that $x_{0} \in K \backslash\{x\}$ and $x-x_{0} \in K^{\infty}$. It follows that $x \notin \operatorname{Min}\left(K, K^{\infty}\right)$. This shows that $\operatorname{Min}\left(K, K^{\infty}\right)=\emptyset$.

It is clear that $K$ has no extreme points if $K$ contains lines. This motivates us to introduce a new concept of what we shall refer to as "generalized extreme points". Let $X$ be a Hilbert space. For a closed convex subset $K$ of $X$, let

$$
\operatorname{Lin}(K)^{\perp}:=\{x \in X:\langle x, y\rangle=0, \quad \forall y \in \operatorname{Lin}(K)\} .
$$

We say that $e$ is a generalized extreme point of $K$ if $e \in K \cap \operatorname{Lin}(K)^{\perp}$ and

$$
x_{1}, x_{2} \in K \text { and } e=\frac{x_{1}+x_{2}}{2} \Longrightarrow x_{1}-x_{2} \in \operatorname{Lin}(K) .
$$

We denote by $\operatorname{ext}_{E}(K)$ the set of all generalized extreme points of $K$. Clearly, $\operatorname{ext}_{E}(K)=\operatorname{ext}(K)$ if $K$ contains no lines (i.e., $\left.\operatorname{Lin}(K)=\{0\}\right)$. Moreover, one has that

$$
\operatorname{ext}_{E}(K) \subset \operatorname{Min}\left(K, K^{\infty}\right)
$$

To see this, let $e \in \operatorname{ext}_{E}(K)$ and $x \in K$ with $x \leq_{K^{\infty}} e$. Then $e-x \in K^{\infty}$ and hence $2 e-x=e+(e-x) \in K$. Since $e=\frac{x+(2 e-x)}{2}$, it follows that $2(e-x) \in \operatorname{Lin}(K)$, which means $x-e \in \operatorname{Lin}(K) \subset K^{\infty}$. Hence $e \leq_{K^{\infty}} x$. This shows that $e \in \operatorname{Min}\left(K, K^{\infty}\right)$. Thus (5.5) is true.

Proposition 5.2. Let $X$ be a Hilbert space and $K$ a closed convex subset of $X$. Then

$$
\operatorname{ext}_{E}(K)=\operatorname{ext}\left(K \cap \operatorname{Lin}(K)^{\perp}\right)
$$

Proof. By definition it is clear that $\operatorname{ext}_{E}(K) \subset \operatorname{ext}\left(K \cap \operatorname{Lin}(K)^{\perp}\right)$. Conversely, let $e \in \operatorname{ext}\left(K \cap \operatorname{Lin}(K)^{\perp}\right)$ and $x_{1}, x_{2} \in K$ satisfy $e=\frac{x_{1}+x_{2}}{2}$. Noting that for each 
$x \in X$ there exists a unique pair $(u, v) \in \operatorname{Lin}(K) \times \operatorname{Lin}(K)^{\perp}$ such that $x=u+v$. Take $\left(u_{1}, v_{1}\right),\left(u_{2}, v_{2}\right) \in \operatorname{Lin}(K) \times \operatorname{Lin}(K)^{\perp}$ such that $x_{1}=u_{1}+v_{1}$ and $x_{2}=u_{2}+v_{2}$. Then $e=\frac{u_{1}+u_{2}}{2}+\frac{v_{1}+v_{2}}{2}$. It follows from $e \in \operatorname{ext}\left(K \cap \operatorname{Lin}(K)^{\perp}\right)$ that $u_{1}+u_{2}=0$ and $e=v_{1}=v_{2}$. Thus, $x_{1}-x_{2}=u_{1}-u_{2} \in \operatorname{Lin}(K)$ and hence $e \in \operatorname{ext}_{E}(K)$. This shows that $\operatorname{ext}_{E}(K) \supset \operatorname{ext}\left(K \cap \operatorname{Lin}(K)^{\perp}\right)$, and (5.6) is proved.

Proposition 5.3. Let $K$ be a closed convex subset of a Hilbert space $X$ and $\prod$ be the project operator to $\operatorname{Lin}(K)^{\perp}$. Suppose that $C$ is a recession core of $K$. Then $\operatorname{ext}_{E}(K) \subset \prod(C)$.

Proof. Let $e \in \operatorname{ext}_{E}(K)$. Then, by (5.1) there exist $x_{1}, \cdots, x_{n} \in C, t_{1}, \cdots, t_{n} \in$ $[0,1]$ with $\sum_{i=1}^{n} t_{i}=1$ and $h \in K^{\infty}$ such that $e=\sum_{i=1}^{n} t_{i} x_{i}+h$. Then,

$$
e=\frac{\left(\sum_{i=1}^{n} t_{i} x_{i}+\frac{1}{2} h\right)+\left(\sum_{i=1}^{n} t_{i} x_{i}+\frac{3}{2} h\right)}{2} .
$$

Thus, by (5.4), one has $h \in \operatorname{Lin}(K)$. It follows from the linearity of $\prod$ that

$$
e=\prod(e)=\prod\left(\sum_{i=1}^{n} t_{i} x_{i}+h\right)=\sum_{i=1}^{n} t_{i} \prod\left(x_{i}\right) \in \prod(C) .
$$

This shows that $\operatorname{ext}_{E}(K) \subset \prod(C)$.

The following proposition shows that $\operatorname{ext}_{E}(K)$ is a recession core of $K$ when $X$ is finite dimensional.

Proposition 5.4. Let $K$ be a closed convex nonempty subset of $R^{n}$. Then

$$
K=\operatorname{co}\left(\operatorname{ext}_{E}(K)\right)+K^{\infty} .
$$

Proof. Let $h \in R^{n}$ be such that $x+R h \subset K \cap \operatorname{Lin}(K)^{\perp}$ for some $x \in R^{n}$. Then $h \in \operatorname{Lin}(K)$, and hence $\langle x+t h, h\rangle=0$ for all $t \in R$. It follows that $h=0$. Therefore $K \cap \operatorname{Lin}(K)^{\perp}$ is a closed convex subset containing no lines. It follows from [27, Theorem 18.5] that

$$
K \cap \operatorname{Lin}(K)^{\perp}=\operatorname{co}\left(\operatorname{ext}\left(K \cap \operatorname{Lin}(K)^{\perp}\right)\right)+\left(K \cap \operatorname{Lin}(K)^{\perp}\right)^{\infty} .
$$

This and Proposition 5.2 imply that $K \cap \operatorname{Lin}(K)^{\perp} \subset \operatorname{co}\left(\operatorname{ext}_{E}(K)\right)+K^{\infty}$. Noting that $K^{\infty}+\operatorname{Lin}(K)=K^{\infty}$, one then has

$$
K \cap \operatorname{Lin}(K)^{\perp}+\operatorname{Lin}(K) \subset \operatorname{co}\left(\operatorname{ext}_{E}(K)\right)+K^{\infty} .
$$

Let $x \in K$ and take $x_{1} \in \operatorname{Lin}(K)$ and $x_{2} \in \operatorname{Lin}(K)^{\perp}$ such that $x=x_{1}+x_{2}$. Hence $x_{2} \in$ $x+\operatorname{Lin}(K) \subset K$ and so $x_{2} \in K \cap \operatorname{Lin}(K)^{\perp}$. Therefore, $K \subset \operatorname{Lin}(K)+K \cap \operatorname{Lin}(K)^{\perp}$. It follows from (5.8) that

$$
K \subset \operatorname{co}\left(\operatorname{ext}_{E}(K)\right)+K^{\infty} .
$$


Thus (5.7) holds as the converse inclusion is obvious.

Remark. Proposition 5.4 shows that $\operatorname{ext}_{E}(K)$ is a recession core of $K$, and has the minimality property "up to $\operatorname{Lin}(K)$ " in the sense as indicated in Proposition 5.3. In particular, if $K$ contains no lines then $\operatorname{ext}_{E}(K)=\operatorname{ext}(K)$ is the least recession core of $K$.

In terms of recession cores and the BCQs, we now study the global metric subregularity of generalized equation (GEC). Hoffman, in his pioneering work, proved that (GEC) has an error bound (or equivalently, is globally metrically subregular) if $A=X=R^{n}$ and $F(x):=Q x+R_{+}^{n}$ for all $x \in R^{n}$, where $Q$ is a $m \times n$ matrix. The research on error bounds, especially for inequality systems, has attracted the interest of many researchers and there are a vast number of publications reporting progress in this area. For more details, see $[17,18,20,23,24,30,31]$ and a special issue of Mathematical Programming (Vol.88, No.2, 2000). In what follows, we assume that $X, Y$ are general Banach spaces (except explicitly stated otherwise), $F$ is a closed convex multifunction from $X$ to $Y$ and that $A$ is a closed convex subset of $X$. In the case when $A=X$, while the equivalence of (iv) with (v) in the following result is [24, Theorem 3.1], we can now sharpen the result by considering recession cores of $S$.

THEOREM 5.1. Let $C$ be a recession core of the solution set $S$ of (GEC) and $\tau \in[0,+\infty)$. Then the following statements are equivalent.

(i) (GEC) has the strong BCQ at each $x \in C$ with the constant $\tau$.

(ii) (GEC) has the strong BCQ at each $x \in S$ with the constant $\tau$.

(iii) (GEC) is metrically subregular at each point in $C$ with the constant $\tau$.

(iv) (GEC) is metrically subregular at each point in $S$ with the constant $\tau$.

(v) (GEC) is globally metrically subregular with the constant $\tau$.

Proof. (i) $\Rightarrow$ (ii) Let $x \in S$. Since $C$ is a recession cone of $S$, there exist $x_{1}, \cdots, x_{n} \in C, t_{1}, \cdots, t_{n} \in[0,+\infty)$ and $e \in S^{\infty}$ such that

$$
\sum_{i=1}^{n} t_{i}=1 \text { and } x=\sum_{i=1}^{n} t_{i} x_{i}+e
$$

Let $x^{*} \in N(S, x) \cap B_{X^{*}}$. Then $\left\langle x^{*}, \sum_{i=1}^{n} t_{i} x_{i}+e\right\rangle=\max \left\{\left\langle x^{*}, z\right\rangle: z \in S\right\}$. Noting that $\sum_{i=1}^{n} t_{i} x_{i}+R_{+} e \subset S$, it follows that

$$
\left\langle x^{*}, e\right\rangle=0 \text { and }\left\langle x^{*}, \sum_{i=1}^{n} t_{i} x_{i}\right\rangle=\max \left\{\left\langle x^{*}, z\right\rangle: z \in S\right\} .
$$

This implies that for each integer $i \in[1, n]$,

$$
\left\langle x^{*}, x\right\rangle=\left\langle x^{*}, x_{i}\right\rangle=\max \left\{\left\langle x^{*}, z\right\rangle: z \in S\right\},
$$

and hence $x^{*} \in N\left(S, x_{i}\right) \cap B_{X^{*}}$. By (i), one has

$$
x^{*} \in \tau\left(D^{*} F\left(x_{i}, b\right)\left(B_{Y^{*}}\right)+B_{X^{*}} \cap N\left(A, x_{i}\right)\right), i=1, \cdots, n .
$$


It follows from Lemma 3.1 that $x^{*} \in \tau\left(D^{*} F(x, b)\left(B_{Y^{*}}\right)+B_{X^{*}} \cap N(A, x)\right)$. Therefore,

$$
N(S, x) \cap B_{X^{*}} \subset \tau\left(D^{*} F(x, b)\left(B_{Y^{*}}\right)+B_{X^{*}} \cap N(A, x)\right) .
$$

This shows that (ii) holds.

(ii) $\Rightarrow$ (i), (iv) $\Rightarrow$ (iii) and (v) $\Rightarrow$ (iv) are trivial. (iii) $\Rightarrow$ (i) and (ii) $\Rightarrow$ (iv) are consequences of formula (3.6) in Remark 3.1. The proof of (iv) $\Rightarrow(\mathrm{v})$ is similar to that of $[24$, Theorem 3.1]. The proof is completed.

In the special case when $A=X, Y=R, F(x)=[f(x),+\infty)$ for all $x \in X$ and $b=\inf \{f(x): x \in X\}$ with $f$ being a proper lower semicontinuous convex function from $X$ to $R \cup\{+\infty\}$, Burke and Deng [3, Theorem 2.3] proved that (GEC) is globally $\tau$-metrically subregular if and only if

$$
N(S, z) \cap B_{X^{*}} \subset \mathrm{cl}^{*}(\partial f(z)) \text { for all } z \in S .
$$

Since $\operatorname{ext}_{E}(S)$ is a recession core of $S$ if $X=R^{n}$, the following corollary is a consequence of Theorem 5.1.

Corollary 5.1. Let $X=R^{n}$. Then (GEC) is globally metrically subregular if and only there exists $\tau \in(0,+\infty)$ such that (GEC) has the strong BCQ at each generalized extreme point of $S$ with the constant $\tau$.

Similar to the proof of the equivalent relation $(\mathrm{i}) \Leftrightarrow($ ii) in Theorem 5.1 , one can prove the following result.

Proposition 5.5. Let $C$ be a recession core of $S$. Then (GEC) has the BCQ at each point in $C$ if and only if (GEC) has the BCQ at each point in $S$.

As in the finite dimensional case, let us say that a subset $P$ of $X$ is a polyhedron if there exist $x_{n}^{*}, \cdots, x_{n}^{*} \in X^{*}$ and $c_{1}, \cdots, c_{n} \in R$ such that

$$
P=\left\{x \in X:\left\langle x_{i}^{*}, x\right\rangle \leq c_{i}, i=1, \cdots, n\right\} .
$$

It is known that

$$
N(P, x)=\left\{\sum_{i \in I(x)} t_{i} x_{i}^{*}: t_{i} \geq 0, i \in I(x)\right\} \quad \forall x \in P
$$

where $I(x):=\left\{1 \leq i \leq n:\left\langle x_{i}^{*}, x\right\rangle=c_{i}\right\}$. We say that a multifunction $F: X \rightarrow 2^{Y}$ is polyhedral if its graph is a polyhedron in $X \times Y$. If $F$ is polyhedral, it is easy to verify from (5.10) that

$$
N\left(F^{-1}(b), x\right)=D^{*} F(x, b)\left(Y^{*}\right) \quad \forall x \in F^{-1}(b) .
$$

ThEOREM 5.2. Let $C$ be a recession core of the solution set $S$ of (GEC). Suppose that $S$ is a polyhedron in $X$. Then $(G E C)$ is globally metrically subregular if and only if (GEC) has the BCQ at each point in $C$. 
Proof. By Theorem 5.1, it suffices to prove the sufficiency. Suppose that (GEC) has the BCQ at each point in $C$. Then, (GEC) has the BCQ at each point of $S$ (by Proposition 5.5). Since $S$ is a polyhedron, there exist $x_{n}^{*}, \cdots, x_{n}^{*}$ and $c_{1}, \cdots, c_{n} \in R$ such that

$$
S=\left\{x \in X:\left\langle x_{i}^{*}, x\right\rangle \leq c_{i}, i=1, \cdots, n\right\} .
$$

Let $X_{1}:=\left\{x \in X:\left\langle x_{i}^{*}, x\right\rangle=0, i=1, \cdots, n\right\}$. Then $X_{1}$ is a closed subspace of $X$ with finite codimension. Thus, there exists a finite dimensional subspace $X_{2}$ of $X$ such that $X=X_{1}+X_{2}$ and $X_{1} \cap X_{2}=\{0\}$. Let

$$
P:=\left\{z \in X_{2}:\left\langle x_{i}^{*}, z\right\rangle \leq c_{i}, i=1, \cdots, n\right\} .
$$

It is easy to verify that $S=P+X_{1}$ and $P$ is a polyhedron containing no lines in $X_{2}$. By [27, Theorems 18.5 and 19.1], $P=\operatorname{co}(\operatorname{ext}(P))+P^{\infty}$. Hence $S=\operatorname{co}(\operatorname{ext}(P))+P^{\infty}+X_{1}$. Noting that $P^{\infty}+X_{1} \subset(S)^{\infty}$, it follows that $\operatorname{ext}(P)$ is a recession core of $S$. Let $e \in \operatorname{ext}(P)$. Then, by $(5.10), N(S, e)$ is a polyhedron in a finite dimensional subspace of $X^{*}$. It follows from Proposition 3.1 that there exists $\tau_{e} \in(0,+\infty)$ such that (GEC) has the strong BCQ with the constant $\tau_{e}$. Do this for each $e$ in $\operatorname{ext}(P)$ and let $\tau:=\max \left\{\tau_{e}: e \in \operatorname{ext}(P)\right\}$. Then $\tau<+\infty$ because $\operatorname{ext}(P)$ is a finite set (cf. [27, Theorem 19.1]). Hence (GEC) has the strong BCQ at each point of ext $(P)$ with the constant $\tau$. Since $\operatorname{ext}(P)$ is a recession core of $S$, it follows from Theorem 5.1 that (GEC) is globally metrically subregular. The proof is completed.

In view of the proof of Theorem 5.2, one sees that any polyhedron in a Banach space has a recession core consisting of finitely many elements.

Robinson [26] studied the continuity properties of polyhedral multifunctions. In particular, under the finite-dimension assumption, he [26, Corollary] proved that if the graph of $F$ is the union of finitely many polyhedra and $b \in F(X)$ then there exists $\varepsilon, \tau \in[0,+\infty)$ such that

$$
d\left(x, F^{-1}(b)\right) \leq \tau d(b, F(x)) \text { for all } x \in X \text { with } d(b, F(x))<\varepsilon .
$$

This result can be regarded as a generalization of Hoffman's classical error bound theorem. In the setting of Theorem 5.2, $F$ is not required to be polyhedral but merely the solution set $S$ is required to be polyhedral. When $F$ is a convex polyhedral multifunction and $A=X,(5.11)$ implies that generalized equation (GEC) has the BCQ at each $x \in S=F^{-1}(b)$. Hence, in this case, Theorem 5.2 improves the above Robinson's result. But, in the nonconvex case, one cannot use Theorem 5.2 to deduce Robinson's result.

Remark. Let $M: Y \rightarrow 2^{X}$ be a closed convex multifunction and with $(\bar{y}, \bar{x}) \in$ $\operatorname{Gr}(M)$. We say that $M$ is globally calm at $\bar{y}$ over $A$ if there exists $\tau \in(0,+\infty)$ such 
that

$$
d(x, M(\bar{y}) \cap A) \leq \tau(\|y-\bar{y}\|+d(x, A)) \text { for all }(x, y) \in \operatorname{Gr}(M)
$$

Let $C$ be a recession core of $M(\bar{y}) \cap A$. Theorems 5.1 implies that $M$ is globally calm at $\bar{y}$ over $A$ if and only if there exists $\tau \in(0,+\infty)$ such that

$$
N(M(\bar{y}) \cap A, u) \cap B_{X^{*}} \subset \tau\left(D^{*} M^{-1}(u, \bar{y})\left(B_{Y^{*}}\right)+N(A, u) \cap B_{X^{*}}\right) \quad \forall u \in C .
$$

In the case when $M(\bar{y}) \cap A$ is a polyhedron, Theorem 5.2 implies that $M$ is globally calm at $\bar{y}$ over $A$ if and only if

$$
N(M(\bar{y}) \cap A, u)=D^{*} M^{-1}(u, \bar{y})\left(Y^{*}\right)+N(A, u) \quad \forall u \in C .
$$

To end this paper, we provide a procedure to find the generalized extreme points of a polyhedron in a finite dimensional space. Let $a_{1}, \cdots, a_{m} \in R^{n}, c_{1}, \cdots, c_{m} \in R$ and let $P$ denote the polyhedron determined by $a_{i}$ and $c_{i}(i=1, \cdots, m)$, that is,

$$
P=\left\{x \in R^{n}:\left\langle a_{i}, x\right\rangle \leq c_{i}, i=1, \cdots, m\right\} .
$$

For convenience, let $I:=\{1, \cdots, m\}$ and $I(x)=\left\{i \in I:\left\langle a_{i}, x\right\rangle=c_{i}\right\}$ for $x \in P$. Let $\mathcal{M}(I)$ denote the family of all subsets $D$ of $I$ with the property that $\left\{a_{i}: i \in D\right\}$ is a maximal linearly independent subset of $\left\{a_{i}: i \in I\right\}$. Thus elements of $\mathcal{M}(I)$ can be obtained by the Gram-Schmidt Process. For each $D \in \mathcal{M}(I)$, the following linear equation system

$$
\sum_{j \in D}\left\langle a_{i}, a_{j}\right\rangle t_{j}=c_{i}, \quad \forall i \in D
$$

has a unique solution which will be denoted by $\left(\bar{t}_{j}\right)_{j \in D}$; we shall also write $e_{D}$ for $\sum_{j \in D} \bar{t}_{j} a_{j}$. Let

$$
\mathcal{E}(I)=\left\{D \in \mathcal{M}(I):\left\langle a_{i}, e_{D}\right\rangle \leq c_{i}, i \in I \backslash D\right\}
$$

Theorem 5.3. $\operatorname{ext}_{E}(P)=\left\{e_{D}: D \in \mathcal{E}(I)\right\}$.

Proof. Note that $\operatorname{Lin}(P)=\left\{x \in R^{n}:\left\langle a_{i}, x\right\rangle=0, i \in I\right\}$. Hence,

$$
\operatorname{Lin}(P)^{\perp}=\operatorname{span}\left\{a_{i}: i \in I\right\}=\operatorname{span}\left\{a_{i}: i \in D\right\} \quad \forall D \in \mathcal{M}(I),
$$

where $\operatorname{span} A$ denotes the linear hull of $A$. Let $e \in \operatorname{ext}_{E}(P)$ and pick a $D_{0} \subset I(e)$ such that $\left\{a_{i}: i \in D_{0}\right\}$ is a maximal linearly independent subset of $\left\{a_{i}: i \in I(e)\right\}$. We claim that $D_{0} \in \mathcal{M}(I)$. Indeed, if this is not the case, then $\operatorname{span}\left\{a_{i}: i \in I(e)\right\}$ is a proper subspace of $\operatorname{span}\left\{a_{i}: i \in I\right\}$. It follows from the first equality of $(5.12)$ that there exists $h \in \operatorname{Lin}(P)^{\perp} \backslash\{0\}$ such that $\left\langle a_{i}, h\right\rangle=0$ for all $i \in I(e)$. Since $\left\langle a_{i}, e\right\rangle\left\langle c_{i}\right.$ for all $i \in I \backslash I(e)$, there exists $\varepsilon>0$ small enough such that $e \pm \varepsilon h \in P$. Since $e=\frac{e+\varepsilon h+(e-\varepsilon h)}{2}$, it follows from (5.4) that $2 \varepsilon h \in \operatorname{Lin}(P)$. This contradicts 
$h \in \operatorname{Lin}(P)^{\perp} \backslash\{0\}$. Hence $D_{0} \in \mathcal{M}(I)$. Noting that $e \in \operatorname{Lin}(P)^{\perp}$ (by Proposition 5.2), it follows from (5.12) that there exists $\left(\bar{t}_{j}\right)_{j \in D_{0}} \in R^{\left|D_{0}\right|}$ such that $e=\sum_{j \in D_{0}} \bar{t}_{j} a_{j}$, where $\left|D_{0}\right|$ denotes the number of elements of $D_{0}$. It follows from $e \in P$ and $D_{0} \subset I(e)$ that $D_{0} \in \mathcal{E}(I)$ and $e=e_{D_{0}}$. Therefore, $\operatorname{ext}_{E}(P) \subset\left\{e_{D}: D \in \mathcal{E}(I)\right\}$. It remains to show that $\left\{e_{D}: D \in \mathcal{E}(I)\right\} \subset \operatorname{ext}_{E}(P)$. To do this, let $D \in \mathcal{E}(I)$. Then $e_{D} \in P \cap \operatorname{Lin}(P)^{\perp}$ (by (5.12) and the definition of $e_{D}$ ). Let $x_{1}, x_{1} \in P$ satisfy $e_{D}=\frac{x_{1}+x_{2}}{2}$. It follows that $\left\langle a_{i}, x_{1}\right\rangle=\left\langle a_{i}, x_{2}\right\rangle=c_{i}$ for all $i \in D$, and so $\left\langle a_{i}, x_{1}-x_{2}\right\rangle=0$ for all $i \in D$. Since $\left\{a_{i}: i \in D\right\}$ is a maximal linearly independent subset of $\left\{a_{i}: i \in I\right\},\left\langle a_{i}, x_{1}-x_{2}\right\rangle=0$ for all $i \in I$. Hence $x_{1}-x_{2} \in \operatorname{Lin}(P)$. This shows that $e_{D} \in \operatorname{ext}_{E}(P)$. The proof is completed.

Acknowledgements. The authors thank the referees for their helpful comments and for the reference [16].

\section{REFERENCES}

[1] J. P. Aubin and H. Frankowska, Set-Valued Analysis, Birkhauser, Boston, 1990.

[2] J. F. Bonnans and A. Shapiro, Perturbation Analysis of Optimization Problems, Springer, New York, 2000.

[3] J. V. Burke and S. Deng, Weak sharp minima revisited, Part I: Basic theory, Control Cybernetics, 31 (2002), 399-469.

[4] J. V. Burke, M. C. Ferris and M. Qian, On the Clarke subdifferential of the distance function of a closed set, J. Math. Anal. Appl., 166 (1992), pp. 199-213.

[5] F. H. Clarke, Optimization and Nonsmooth Analysis, Wiley, New York, 1983.

[6] A. L. Dontchev, A. S. Lewis, and R. T. Rockafellar, The radius of metric regularity, Trans. Amer. Math. Soc., 355 (2003), pp. 493-517.

[7] A. L. Dontchev and R. T. Rockafellar, Regularity and conditioning of solution mappings in variational analysis, Set-Valued Anal., 12 (2004), pp.79-109.

[8] R. Henrion and A. Jourani, Subdifferential conditions for calmness of convex constraints, SIAM J. Optim., 13 (2002), pp. 520-534.

[9] R. Henrion, A. Jourani and J. Outrata, On the calmness of a class of multifunctions, SIAM J. Optim., 13 (2002), PP. 603-618.

[10] R. Henrion and J. Outrata, Calmness of constraint systems with applications, Math. Program., 104 (2005), pp.437-464.

[11] J.-B. Hiriart-Urruty and C. Lemarechal, Convex Analysis and Minimization Algorithms I, Springer-Verlag, Berlin, Heidelberg, 1993.

[12] H. Hu, Characterizations of the strong basic constraint qualification, Math. Oper. Res., 30 (2005), pp.956-965.

[13] A. D. Ioffe, Metric regularity and subdifferential calculus, Russian Math. Surveys 55 (2000), pp. 501-558.

[14] G. Jameson, Ordered Linear Spaces, Springer-Verlag, Berlin, 1970.

[15] D. Klatte and B. Kummer, Nonsmooth Equations in Optimization. Regularity, Calculus, Methods and Applications, Nonconvex Optimization and its Application 60, Kluwer Academic Publishers, Dordrecht, 2002.

[16] D. Klatte and B. Kummer, Constraint minima and Lipschitzian penalties in metric spaces, SIAM J. Optim., 13 (2002), pp.619-633.

[17] A. Lewis and J. S. Pang, Error bounds for convex inequality systems, in Generalized Convexity, Generalized Monotonicity: Recent Results, Proceedings of the Fifth Symposium on 
Generalized Convexity, Luminy, June 1996, J.-P. Crouzeix, J.-E. Martinez-Legaz, and M. Volle, eds., Kluwer Academic Publishers, Dordrecht, 1997, pp. 75-100.

[18] W. Li, Abadie's constraint qualification, metric regularity, and error bounds for differentiable convex inequalities, SIAM J. Optim., 7 (1997), pp.966-978.

[19] W. Li, C. Nahak, and I. Singer, Constraint qualifications for semi-infinite systems of convex inequalities, SIAM J. Optim., 11(2000), pp.31-52.

[20] W. Li and I. Singer, Global error bounds for convex multifunctions and applications, Math. Oper. Res., 23 (1998), pp.443-462.

[21] B. S. Mordukhovich, Complete characterization of openness, metric regularity, and Lipschitzian properties of multifunctions, Trans. Amer. Math. Soc., 340 (1994), pp.1-35.

[22] B. S. Mordukhovich and Y. Shao, Nonconvex differential calculus for infinite-dimensional multifunctions, Set-Valued Anal., 4 (1996), pp. 205-236.

[23] K. F. Ng and W. H. Yang, Error bound of abstract linear system, SIAM J. Optim., 13 (2002), pp. $24-43$.

[24] K. F. Ng and X. Y. Zheng, Characterizations of error bounds for convex multifunctions on Banach spaces, Math. Oper. Res., 29 (2004), pp.45-63.

[25] S. M. Robinson, Normed convex processes, Trans. Amer. Math. Soc., 174 (1972), pp. 127-140.

[26] S. M. Robinson, Some continuity properties of polyhedral multifunctions, Math. Program. Studies, 14 (1981), pp. 206-214.

[27] R. T. Rockafellar, Convex Analysis, Princeton University Press, Princeton, New Jersey, 1970.

[28] R. T. Rockafellar and R. J.-B. Wets, Variational Analysis, Springer-Verlag, Berlin, 1998.

[29] C. Ursescu, Multifunctions with closed, convex graph, Czechoslovak Math. J., 25 (1975), pp. 438-441.

[30] C. Zalinescu, A nonlinear extension of Hoffman's error bounds for linear inequalities, Math. Oper. Res., 28 (2003), pp.524-532.

[31] C. Zalinescu, Weak sharp minima, well-behaving functions and global error bounds for convex inequalities in Banach spaces, Proc. 12th Baical Internat. Conf. on Optimization Methods and their applications, Irkutsk, Russia, 2001, pp.272-284.

[32] X. Y. Zheng and K. F. Ng, Metric regularity and constraint qualifications for convex inequalities on Banach spaces, SIAM J. Optim., 14 (2003), pp.757-772. 